\title{
Iterating Octagons
}

\author{
Marius Bozga, Codruţa Gîrlea, and Radu Iosif \\ VERIMAG/CNRS, 2 Avenue de Vignate, 38610 Gières, France \\ \{bozga,girlea,iosif\}@imag.fr
}

\begin{abstract}
In this paper we prove that the transitive closure of a nondeterministic octagonal relation using integer counters can be expressed in Presburger arithmetic. The direct consequence of this fact is that the reachability problem is decidable for flat counter automata with octagonal transition relations. This result improves the previous results of Comon and Jurski [7] and Bozga, Iosif and Lakhnech [6] concerning the computation of transitive closures for difference bound relations. The importance of this result is justified by the wide use of octagons to computing sound abstractions of real-life systems 15 . We have implemented the octagonal transitive closure algorithm in a prototype system for the analysis of counter automata, called FLATA, and we have experimented with a number of test cases.
\end{abstract}

\section{Introduction}

Counter automata (register machines) are widely investigated models of computation. Since the result of Minsky [16] showing Turing-completeness of 2-counter machines, research on counter automata pursued in two directions. The first one is defining subclasses of counter automata for which various decision problems (e.g. reachability, emptiness, boundedness, disjointness, containment, equivalence) are found to be decidable. Examples include Reversal-bounded Counter Machines [13, Petri Nets and Vector Addition Systems [18] or Flat Counter Automata 14. Often, decidability of various problems is achieved by defining the set of reachable configurations in a decidable logic, such as Presburger arithmetic [17. Such definitions are precise, i.e. no information is lost by the use of over-approximation.

Another, orthogonal, direction of work is concerned with finding sound (but not necessarily complete) answers to the decision problems mentioned above, in a cost-effective way. Such approaches use abstract domains (Polyhedra [8], Octagons [15, Difference Constraints [1, etc.) and compute fixed points that are over-approximations of the set of reachable configurations.

Both approaches to the analysis of counter automata benefit, in some sense, from algorithms for computing transitive closures of arithmetic relations. If $I$ is the initial set of configurations, and $R$ is the transition relation of the counter automaton, then $R^{*}(I)$ (the image of $I$ through $R^{*}$ ) is the set of reachable configurations, where $R^{*}=\bigcup_{n \geq 0} R^{n}$ is the transitive closure of $R$. The problem lies essentially in expressing the infinite disjunction from the definition of $R^{*}$, in a finite way.

S. Kowalewski and A. Philippou (Eds.): TACAS 2009, LNCS 5505, pp. 337-351 2009.

(C) Springer-Verlag Berlin Heidelberg 2009 
In this paper we consider octagonal transition relations that are conjunctions of atoms of the form $\pm x \pm y \leq c$, where $x$ and $y$ are counter values, either at the current step, or at the next step (in which case they are denoted by primed variables), and $c \in \mathbb{Z}$ is an integer constant. For this class of relations, we prove that the transitive closure is expressible in Presburger arithmetic [17. This improves the previous result of Comon and Jurski [7], showing that the transitive closure of a difference bound constraint (a conjunction of atoms of the form $x-y \leq c$, with $x, y$ possible primed counters, and $c \in \mathbb{Z}$ ) is Presburgerdefinable.

We adopt the classical representation of octagonal constraints (or octagons) $\varphi\left(x_{1}, \ldots, x_{n}\right)$ as difference bound constraints $\varphi\left(y_{1}, \ldots, y_{2 n}\right)$, where $y_{2 i-1}$ stands for $+x_{i}$ and $y_{2 i}$ for $-x_{i}$, and the implicit condition $y_{2 i-1}+y_{2 i}=0,1 \leq i \leq n$. With this convention, 2] provides an algorithm for computing the canonical form of an octagon, by first computing the strongest closure of the corresponding difference bound constraint (using the classical Floyd-Warshall cubic algorithm), and subsequently tightening the constraints of the form $y_{2 i}-y_{2 i-1} \leq c$ and $y_{2 i-1}-y_{2 i} \leq d$ by adjusting $c$ to $2\lfloor c / 2\rfloor$ and $d$ to $2\lfloor d / 2\rfloor$, respectively $\sqrt{1}$. We apply this idea to tightening octagonal relations of the form $R^{k}$, where $R$ is an octagon and $k \geq 1$ is the arbitrary number of iterations, obtaining in this way a Presburger formula $\psi\left(k, x_{1}, \ldots, x_{n}, x_{1}^{\prime}, \ldots, x_{n}^{\prime}\right)$ equivalent to the $k$-th iteration of $\varphi$. The transitive closure of $R$ is thus $R^{*}=\exists k . \psi$.

The main application of this result is that the following problems are decidable, for the class of counter automata with octagonal transition rules:

- reachability: the automaton has a run leading from a configuration in $I$ to a configuration in $S$, where $I$ and $S$ are Presburger-definable sets of configurations.

- emptiness: the automaton has at least a run starting with all counters set to zero, and leading to a final control location.

In particular, decidability of the reachability problem is useful for the verification of safety properties (e.g. assertion checking) of integer programs, whereas the emptiness problem is a promising approach for the analysis of programs with integer arrays. Indeed, the works of [115] reduce the satisfiability problem for two logics on integer arrays to the emptiness problem of a counter automaton. By enlarging the class of counter automata for which this problem is decidable, we enhance the expressiveness of (decidable) logics of integer arrays.

Finally, we have implemented our method in a tool for the analysis of counter automata, called FLATA 10. In particular, FLATA computes the transitive closure of elementary octagonal cycles, which is used in computing the set of reachable configurations for flat counter automata. We have experimented with a number of test cases and provided several experimental results.

${ }^{1}$ This is needed because we consider the counters to range over integer numbers. If rationals or real number would have been used, this would not have been needed. 


\subsection{Related Work}

The domain of counter machines has been investigated starting with the seminal work of Minsky [16]. His result on Turing-completeness of 2-counter machines motivated research on subclasses of counter automata, for which some problems are decidable, such as: Reversal-bounded Counter Machines [13, Petri Nets and Vector Addition Systems [18] or Flat Counter Automata 14].

The class of Flat Counter Automata which is closest to our work is the one studied by Comon and Jurski in [7]. In this work, they prove that the transitive closure of a difference bound constraint is Presburger-definable. In [6] we showing how to effectively compute the transitive closure of a difference bound constraint directly as a finite set of linear inequation systems, opening thus the possibility of using SMT tools for the analysis of such models.

Octagonal constraints are investigated in the comprehensive paper of Miné 15. Among other results, his paper presents a cubic time tightening algorithm for an octagonal constraint, which is an improvement of the classical algorithm of Harvey and Stuckey [12. However, this algorithm is not suitable to tightening octagonal constraints of parametric size, as the ones we obtain by iteration. For this reason, we adapt the (newer) tightening algorithm of Bagnara, Hill and Zaffanella [2] to octagons obtained by arbitrary iteration, as they prove that it is sufficient to adjust the constants after the computation of minimal paths (by the Floyd-Warshall algorithm).

Our paper extends to octagons the class of non-deterministic relations for which the transitive closure can be effectively expressed in Presburger arithmetic. This result relies essentially on our method from [6] to computing minimal paths in constraint graphs obtained by iteration, and the idea of [2] for tightening (finite) octagonal relations.

On a different line of work, Boigelot [3, and Finkel and Leroux [9] have studied the computation of transitive closure for affine relations of the form $\mathbf{x}^{\prime}=A \mathbf{x}+\mathbf{b}$. Their class of systems differs in nature from ours, in what their transition relations are functional (deterministic), whereas octagons are not. Because of this, their results seem incomparable to ours.

Roadmap. The paper is organized as follows. Section 2 introduces difference bound constraints and difference bound relations, recalling a number of results on DBMs, Section 3 defines octagonal constraints, and gives a necessary quantifier elimination result on octagons, while Section 4 is dedicated to our main result, namely computing the transitive closure of an octagonal relation. Section 5 gives implementation results, and finally, Section 6 concludes. Due to space reasons, all proofs are given in [4].

\section{Difference Bounds}

In this section we recall several definitions and results on difference bound constraints, and the transitive closure of difference bound relations. In the rest of the paper, $\mathbb{Z}$ denotes the set of integer numbers, $n>0$ is the number of variables 
and $\mathbf{x}=\left(x_{1}, x_{2}, \ldots, x_{n}\right)$ is the tuple of variables. If $\varphi(\mathbf{x})$ is a logical formula in which $x_{1}, x_{2}, \ldots, x_{n}$ occur free, and $\mathbf{v}=\left(v_{1}, v_{2}, \ldots, v_{n}\right)$ is a tuple of values, $\varphi[\mathbf{v} / \mathbf{x}]$ denotes the formula in which each occurrence of $x_{i}$ has been replaced by $v_{i}$. We denote by $\mathbf{v}=\varphi$ the fact that $\varphi[\mathbf{v} / \mathbf{x}]$ is logically equivalent to true. We say that $\varphi$ is consistent if there exists at least one $\mathbf{v} \in \mathbb{Z}^{n}$ such that $\mathbf{v}=\varphi$, and inconsistent otherwise. If $\varphi$ is a formula, let $A P(\varphi)$ denote the set of atomic propositions in $\varphi$. If $m$ is a matrix, then $m_{i, j}$ denote the element of $m$ situated at line $i$ and column $j$.

\subsection{Difference Bound Constraints}

Definition 1. Let $\mathbf{x}=\left(x_{1}, x_{2}, \ldots, x_{n}\right)$ be a set of variables ranging over $\mathbb{Z}$. Then a formula $\phi(\mathbf{x})$ is a difference bound constraint if it is equivalent to a finite conjunction of atomic propositions of the form $x_{i}-x_{j} \leq \alpha_{i, j}, i \neq j, 1 \leq i, j \leq n$, where $\alpha_{i, j} \in \mathbb{Z}$.

For instance, $x-y=5$ is a difference bound constraint, as it is equivalent to $x-y \leq 5 \wedge y-x \leq-5$. In practice difference bound constraints are represented either as matrices or as graphs, each of these representations being suitable for particular procedures (e.g. closure, iteration). We define these representations and procedures below.

Definition 2. Let $\mathbf{x}=\left(x_{1}, x_{2}, \ldots, x_{n}\right)$ be a set of variables ranging over $\mathbb{Z}$ and $\phi(\mathbf{x})$ be a difference bound constraint. Then a difference bound matrix (DBM) representing $\phi$ is an $n \times n$ matrix $m$ such that:

$$
m_{i, j}= \begin{cases}\alpha_{i, j} & \text { if }\left(x_{i}-x_{j} \leq \alpha_{i, j}\right) \in A P(\phi) \\ \infty & \text { otherwise }\end{cases}
$$

For a $n \times n$ DBM $m$ we denote by $\gamma(m)=\left\{\mathbf{v} \in \mathbb{Z}^{n} \mid v_{i}-v_{j} \leq m_{i, j}, 1 \leq i, j \leq n\right\}$ the set of concretizations of $m$. Notice that this is exactly the set of models of the corresponding difference bound constraint $\varphi$, i.e. $\gamma(m)=\{\mathbf{v} \mid \mathbf{v}=\varphi\}$. A DBM $m$ is said to be consistent if $\gamma(m) \neq \emptyset$, and inconsistent otherwise.

Definition 3. Let $\mathbf{x}=\left(x_{1}, x_{2}, \ldots, x_{n}\right)$ be a set of variables ranging over $\mathbb{Z}$ and $\phi(\mathbf{x})$ be a difference bound constraint. Then $\phi$ can be represented as a weighted graph $\mathcal{G}$ with vertices $x_{1}, x_{2}, \ldots, x_{n}$ in which there is an arc with weight $\alpha_{i, j}$ between $x_{i}$ and $x_{j}$ in $\mathcal{G}$ if there is a constraint $x_{i}-x_{j} \leq \alpha_{i, j}$ in $\phi$. This graph is also called a constraint graph.

Whenever the graph $\mathcal{G}$ is obvious from the context, we will denote by $x \stackrel{\alpha}{\rightarrow} y$ the fact that there exists an edge with weight $\alpha$ from $x$ to $y$ in $\mathcal{G}$. Notice that the DBM $m$ of a difference bound constraint is the incidence matrix of its corresponding constraint graph. This graph will be denoted as $\mathcal{G}(m)$ in the following. The three notions (constraint, matrix, graph) are related by the following property: 
Property 1. Let $\phi$ be a difference bound constraint, $m$ the corresponding DBM and $\mathcal{G}(m)$ the corresponding graph. Then the following three statements are equivalent :

1. $\phi$ is inconsistent

2. $\mathcal{G}(m)$ contains at least one negative weight cycle

3. $\gamma(m)=\emptyset$

On one hand, the DBM representation of a difference bound constraint is suitable for computing its normal form, i.e. the most "explicit" formula that has the same set of models.

Definition 4. An $n \times n$ consistent DBM $m$ is said to be closed if and only if the following hold:

1. $m_{i, i}=0 \forall 1 \leq i \leq n$

2. $m_{i, j} \leq m_{i, k}+m_{k, j} \forall 1 \leq i, j, k \leq n$

The shortest path closure of a consistent DBM $m$ is a closed DBM $m^{*}$ such that $\gamma(m)=\gamma\left(m^{*}\right)$. It is well-known that, if $m$ is consistent then $m^{*}$ is unique, and it can be computed from $m$ in time $\mathcal{O}\left(n^{3}\right)$, by the classical Floyd-Warshall algorithm. Moreover, the following condition holds, for all $1 \leq i, j \leq n$ :

$$
m_{i, j}^{*}=\min \left\{\sum_{l=0}^{k-1} m_{i_{l}, i_{l+1}} \mid x_{i}=x_{i_{0}} x_{i_{1}} \ldots x_{i_{k}}=x_{j} \text { is a path in } \mathcal{G}(m)\right\}
$$

On the other hand, the graph representation of a difference bound constraint is suitable for existential quantifier elimination 2 . Concretely, given a difference bound constraint $\phi(\mathbf{x})$, the formula $\exists x_{k} . \phi$ is a difference bound constraint as well, and its corresponding graph is effectively computable from the graph of $\phi$, as shown by the following property :

Property 2. Let $\mathbf{x}=\left(x_{1}, x_{2}, \ldots, x_{n}\right)$ be a set of variables ranging over $\mathbb{Z}, \phi(\mathbf{x})$ be a difference bound constraint and $m$ be the corresponding $n \times n$ DBM. Then, for any $1 \leq k \leq n$ the formula $\exists x_{k} . \phi(\mathbf{x})$ is a difference bound constraint, and its corresponding constraint graph is obtained by erasing the vertex $x_{k}$ together with the incident arcs from the graph $\mathcal{G}\left(m^{*}\right)$. Moreover, the DBM of the resulting graph is also closed.

The importance of this result will be made clear in the next section, because it directly implies that the class of difference bound relations is closed under composition. This is the first ingredient of our transitive closure method.

${ }^{2}$ In general, difference bound constraints are not closed under universal quantification, however finite disjunctions of difference bound constraints are, since the negation of a difference bound constraint is always equivalent to a finite disjunction of difference constraints. 


\subsection{Difference Bound Relations}

Definition 5. Let $\mathbf{x}=\left(x_{1}, x_{2}, \ldots, x_{n}\right), \mathbf{x}^{\prime}=\left(x_{1}^{\prime}, x_{2}^{\prime}, \ldots, x_{n}^{\prime}\right)$ be sets of variables ranging over $\mathbb{Z}$. A relation $R\left(\mathbf{x}, \mathbf{x}^{\prime}\right)$ is a difference bound relation if it is equivalent to a finite conjunction of terms of the form $x_{i}-x_{j} \leq a_{i, j}, x_{i}^{\prime}-x_{j} \leq b_{i, j}$, $x_{i}-x_{j}^{\prime} \leq c_{i, j}$, or $x_{i}^{\prime}-x_{j}^{\prime} \leq d_{i, j}$, where $a_{i, j}, b_{i, j}, c_{i, j}, d_{i, j} \in \mathbb{Z}, 1 \leq i, j \leq n$.

According to the previous section, a difference bound relation can be represented as a constraint graph with nodes in the set $\mathbf{x} \cup \mathbf{x}^{\prime}$, such that $x \stackrel{\alpha}{\rightarrow} y$ if and only if $x-y \leq \alpha \in A P(R)$, for all $x, y \in \mathbf{x} \cup \mathbf{x}^{\prime}$. Just like before, the incidence matrix of this graph is the DBM of the relation.

In the following, we denote $\mathbf{x}^{(l)}=\left(x_{1}^{(l)}, x_{2}^{(l)}, \ldots, x_{n}^{(l)}\right), \mathbf{x}^{(\geq l)}=\bigcup_{s \geq l} \mathbf{x}^{(s)}$ and $\mathbf{x}^{(\leq l)}=\bigcup_{s \leq l} \mathbf{x}^{(s)}$, for any $l \geq 0$. Given a difference bound relation $R\left(\mathbf{x}, \mathbf{x}^{\prime}\right)$, we define the $k$-th iteration of $R$ :

$$
R^{k}\left(\mathbf{x}^{(0)}, \mathbf{x}^{(k)}\right)=\exists \mathbf{x}^{(1)} \ldots \exists \mathbf{x}^{(k-1)} \cdot R\left(\mathbf{x}^{(0)}, \mathbf{x}^{(1)}\right) \wedge \ldots \wedge R\left(\mathbf{x}^{(k-1)}, \mathbf{x}^{(k)}\right)
$$

for all $k \geq 1$. The transitive closure of $R$ is defined as $R^{*}=\exists k \cdot R^{k}$.

Notice that, since existential quantifiers can be eliminated from difference bound constraints (cf. Theorem 2), difference bound relations are closed under composition, and therefore $R^{k}$ is a difference bound relation, for any $k \geq 0$.

We now recall a result from [6], namely that the $k$-th iteration $R^{k}$ of a difference bound relation $R$ is equivalent to a finite set of linear inequation systems. As a result, $R^{*}$ is directly definable in Presburger arithmetic.

The constraint graph $\mathcal{G}^{k}$, representing the matrix of $R^{k}\left(\right.$ i.e. $R\left(\mathbf{x}^{(0)}, \mathbf{x}^{(1)}\right) \wedge \ldots \wedge$ $\left.R\left(\mathbf{x}^{(k-1)}, \mathbf{x}^{(k)}\right)\right)$ is the graph composed of $k$ connected copies of the constraint graph of $R$.

Definition 6. Let $\mathbf{x}=\left(x_{1}, x_{2}, \ldots, x_{n}\right), \mathbf{x}^{\prime}=\left(x_{1}^{\prime}, x_{2}^{\prime}, \ldots, x_{n}^{\prime}\right)$ be sets of variables ranging over $\mathbb{Z}$ and $R\left(\mathbf{x}, \mathbf{x}^{\prime}\right)$ a DBM relation. The constraint graph of $R^{k}$ has as set of vertices $\bigcup_{l=0}^{k} \mathbf{x}^{(l)}$ and, for all $1 \leq i, j \leq n$, for all $0 \leq l<k$ :

$$
\begin{aligned}
x_{i}^{(l)} \stackrel{a}{\rightarrow} x_{j}^{(l)} & \Longleftrightarrow x_{i}-x_{j} \leq a \in A P(R) \\
x_{i}^{(l)} \stackrel{a}{\rightarrow} x_{j}^{(l+1)} & \Longleftrightarrow x_{i}-x_{j}^{\prime} \leq a \in A P(R) \\
x_{i}^{(l+1)} \stackrel{a}{\rightarrow} x_{j}^{(l)} & \Longleftrightarrow x_{i}^{\prime}-x_{j} \leq a \in A P(R) \\
x_{i}^{(l+1)} \stackrel{a}{\rightarrow} x_{j}^{(l+1)} & \Longleftrightarrow x_{i}^{\prime}-x_{j}^{\prime} \leq a \in A P(R)
\end{aligned}
$$

In order to compute $R^{k}$, for a given $k \geq 1$, we must first compute the closure of the constraint graph from definition 6 , in order to remove the intermediary nodes $\mathbf{x}^{(l)}, 0<l<k$. According to Theorem 2, $R^{k}$ is a difference bound constraint, and the graph obtained after eliminating the intermediary variables is the constraint graph of $R^{k}$. Therefore, $R^{k}$ can be written as a conjunction of the form: 


$$
\begin{gathered}
\bigwedge_{1 \leq i, j \leq n} x_{i}^{(0)}-x_{j}^{(0)} \leq \min _{k}\left\{x_{i}^{(0)} \leadsto x_{j}^{(0)}\right\} \wedge \bigwedge_{1 \leq i, j \leq n} x_{i}^{(0)}-x_{j}^{(k)} \leq \min _{k}\left\{x_{i}^{(0)} \leadsto x_{j}^{(k)}\right\} \wedge \\
\bigwedge_{1 \leq i, j \leq n} x_{i}^{(k)}-x_{j}^{(k)} \leq \min _{k}\left\{x_{i}^{(k)} \leadsto x_{j}^{(k)}\right\} \wedge \bigwedge_{1 \leq i, j \leq n} x_{i}^{(k)}-x_{j}^{(0)} \leq \min _{k}\left\{x_{i}^{(k)} \leadsto x_{j}^{(0)}\right\}
\end{gathered}
$$

where $\min _{k}\{x \sim y\} \in \mathbb{Z} \cup\{ \pm \infty\}$ denotes the value of the minimal path between nodes $x$ and $y$ in the constraint graph of $R^{k} 3$. Notice that $R^{k}$ is satisfiable if and only there are no cycles of negative cost within the constraint graph from Definition 6 (cf. property 11). Since this graph is obtained by connecting $k$ copies of $R$, if there is a negative cycle in the graph, there will also be a negative cycle that goes through $x_{i}^{(0)}$, for some $1 \leq i \leq n$.

The main result of [7]6] is that $\min _{k}\left\{x_{i}^{(0)} \leadsto x_{j}^{(0)}\right\}, \min _{k}\left\{x_{i}^{(0)} \leadsto x_{j}^{(k)}\right\}$, $\min _{k}\left\{x_{i}^{(k)} \leadsto x_{j}^{(k)}\right\}$ and $\min _{k}\left\{x_{i}^{(k)} \leadsto x_{j}^{(0)}\right\}$ are Presburger definable functions.
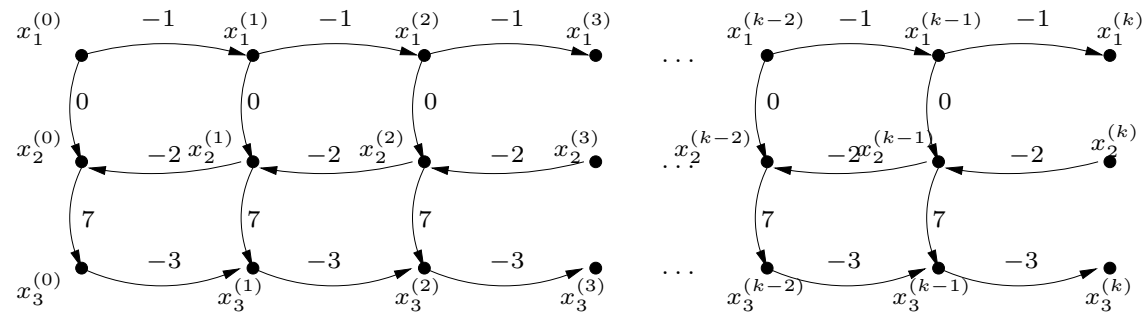

Fig. 1. Graph representation of $R^{k}, k=1,2,3, \ldots$

Example 1. Let $R\left(\mathbf{x}, \mathbf{x}^{\prime}\right)$ be the following DBM relation, where $\mathbf{x}=\left\{x_{1}, x_{2}, x_{3}\right\}$ : $R\left(\mathbf{x}, \mathbf{x}^{\prime}\right)=x_{1}-x_{1}^{\prime} \leq-1 \wedge x_{1}-x_{2} \leq 0 \wedge x_{2}-x_{3} \leq 7 \wedge x_{2}^{\prime}-x_{2} \leq-2 \wedge x_{3}-x_{3}^{\prime} \leq-3$

Figure 1 shows the graph representation of $R^{k}$, for $k=1,2,3 \ldots$ By computing the minimal weight paths in the constraint graph of $R^{k}$ for $k=1,2,3, \ldots$ we obtain, e.g. :

$$
\begin{aligned}
R^{(1)}\left(\mathbf{x}, \mathbf{x}^{\prime}\right)= & x_{1}-x_{2} \leq 0 \wedge x_{1}-x_{3} \leq 7 \wedge x_{2}-x_{3} \leq 7 \wedge x_{1}-x_{1}^{\prime} \leq-1 \wedge x_{1}-x_{3}^{\prime} \leq 4 \wedge \\
& x_{2}-x_{3}^{\prime} \leq 4 \wedge x_{3}-x_{3}^{\prime} \leq-3 \wedge x_{2}^{\prime}-x_{2} \leq-2 \wedge x_{2}^{\prime}-x_{3} \leq 5 \wedge x_{2}^{\prime}-x_{3}^{\prime} \leq 2 \\
R^{(2)}\left(\mathbf{x}, \mathbf{x}^{\prime}\right)= & x_{1}-x_{2} \leq-3 \wedge x_{1}-x_{3} \leq 4 \wedge x_{2}-x_{3} \leq 7 \wedge x_{1}-x_{1}^{\prime} \leq-2 \wedge x_{1}-x_{3}^{\prime} \leq-2 \wedge \\
& x_{2}-x_{3}^{\prime} \leq 1 \wedge x_{3}-x_{3}^{\prime} \leq-6 \wedge x_{2}^{\prime}-x_{2} \leq-4 \wedge x_{2}^{\prime}-x_{3} \leq 3 \wedge x_{2}^{\prime}-x_{3}^{\prime} \leq-3 \\
R^{(3)}\left(\mathbf{x}, \mathbf{x}^{\prime}\right)= & x_{1}-x_{2} \leq-6 \wedge x_{1}-x_{3} \leq 1 \wedge x_{2}-x_{3} \leq 7 \wedge x_{1}-x_{1}^{\prime} \leq-3 \wedge x_{1}-x_{3}^{\prime} \leq-8 \wedge \\
& x_{2}-x_{3}^{\prime} \leq-2 \wedge x_{3}-x_{3}^{\prime} \leq-9 \wedge x_{2}^{\prime}-x_{2} \leq-6 \wedge x_{2}^{\prime}-x_{3} \leq 1 \wedge x_{2}^{\prime}-x_{3}^{\prime} \leq-8 \\
R^{(n \geq 4)}\left(\mathbf{x}, \mathbf{x}^{\prime}\right)= & x_{1}-x_{2} \leq-3-3(n-2) \wedge x_{1}-x_{3} \leq-2-3(n-4) \wedge x_{2}-x_{3} \leq 7 \wedge \\
& x_{1}-x_{1}^{\prime} \leq-n \wedge x_{1}-x_{3}^{\prime} \leq-14-6(n-4) \wedge x_{2}-x_{3}^{\prime} \leq 1-3(n-2) \wedge \\
& x_{3}-x_{3}^{\prime} \leq-3 n \wedge x_{2}^{\prime}-x_{2} \leq-2 n \wedge x_{2}^{\prime}-x_{3} \leq 3-2(n-2) \wedge \\
& x_{2}^{\prime}-x_{3}^{\prime} \leq-3-5(n-2)
\end{aligned}
$$

${ }^{3}$ If $\min _{k}\{x \leadsto y\}=-\infty$ the constraint is logically equivalent to false, and if $\min \{x \leadsto$ $y\}=\infty$ it is logically equivalent to true. 


\section{Octagonal Constraints}

This section is dedicated to octagonal constraints. We provide preliminary results that are needed to define transitive closures of octagonal relations, in the next section.

Definition 7. Let $\mathbf{x}=\left(x_{1}, x_{2}, \ldots, x_{n}\right)$ be a set of variables ranging over $\mathbb{Z}$. Then a formula $\phi(\mathbf{x})$ is an octagonal constraint if it is equivalent to a finite conjunction of terms of the form $\pm x_{i} \pm x_{j} \leq \alpha_{i, j}, 2 x_{i} \leq \beta_{i}$, or $-2 x_{i} \leq \delta_{i}$, where $\alpha_{i, j}, \beta_{i}, \delta_{i} \in \mathbb{Z}$ and $i \neq j, 1 \leq i, j \leq n$.

The name octagon comes from the fact that, in two dimensions, these constraints can be graphically represented by polyhedra with at most eight edges. We represent octagons using the set of variables $\mathbf{y}=\left(y_{1}, y_{2}, \ldots, y_{2 n}\right)$, with the convention that $y_{2 i-1}$ stands for $x_{i}$ and $y_{2 i}$ for $-x_{i}$, respectively. For instance, the octagonal constraint $x_{1}+x_{2}=3$ is represented as $y_{1}-y_{4} \leq 3 \wedge y_{2}-y_{3} \leq-3$.

If we denote by $\bar{\phi}=\phi\left[y_{1} / x_{1}, y_{2} /-x_{1}, \ldots, y_{2 n-1} / x_{n}, y_{2 n} /-x_{n}\right]$, we obtain the following valid entailment: $\phi(\mathbf{x}) \rightarrow\left(\exists y_{2}, y_{4}, \ldots, y_{2 n} . \bar{\phi}\right)\left[x_{1} / y_{1}, \ldots, x_{n} / y_{2 n-1}\right]$. Moreover $\phi(\mathbf{x}) \leftrightarrow\left(\exists y_{2}, y_{4}, \ldots, y_{2 n} . \bar{\phi} \wedge \bigwedge_{i=1}^{n} y_{2 i-1}+y_{2 i}=0\right)\left[x_{1} / y_{1}, \ldots, x_{n} / y_{2 n-1}\right]$.

To handle the $\mathbf{y}$ variables in the following, we define $\bar{i}=i-1$, if $i$ is even, and $\bar{i}=i+1$ if $i$ is odd. Obviously, we have $\overline{\bar{i}}=i$, for all $i \in \mathbb{Z}, i \geq 0$. The following definition extends the matrix representation from difference bound constraints to octagons:

Definition 8. Let $\mathbf{x}=\left(x_{1}, x_{2}, \ldots, x_{n}\right)$ be a set of variables ranging over $\mathbb{Z}$ and $\phi(\mathbf{x})$ be an octagonal constraint. Then an octagonal difference bound matrix or octagonal DBM representing $\phi$ is an $2 n \times 2 n$ matrix $m$ such that:

$$
\begin{aligned}
& \left(x_{i}-x_{j} \leq \alpha_{i, j}\right) \in A P(\phi) \Longleftrightarrow m_{2 i-1,2 j-1}=m_{2 j, 2 i}=\alpha_{i, j} \\
& \left(-x_{i}-x_{j} \leq \alpha_{i, j}\right) \in A P(\phi) \Longleftrightarrow m_{2 i, 2 j-1}=m_{2 j, 2 i-1}=\alpha_{i, j} \\
& \left(-x_{i}+x_{j} \leq \alpha_{i, j}\right) \in A P(\phi) \Longleftrightarrow m_{2 i, 2 j}=m_{2 j-1,2 i-1}=\alpha_{i, j} \\
& \left(x_{i}+x_{j} \leq \alpha_{i, j}\right) \in A P(\phi) \Longleftrightarrow m_{2 i-1,2 j}=m_{2 j-1,2 i}=\alpha_{i, j} \\
& \left(2 x_{i} \leq \beta_{i}\right) \in A P(\phi) \quad \Longleftrightarrow m_{2 i-1,2 i}=\beta_{i} \\
& \left(-2 x_{i} \leq \delta_{i}\right) \in A P(\phi) \quad \Longleftrightarrow m_{2 i, 2 i-1}=\delta_{i}
\end{aligned}
$$

A $2 n \times 2 n$ octagonal DBM $m$ is said to be coherent if and only if $m_{i, j}=m_{\bar{j}, \bar{i}}$, for all $1 \leq i, j \leq 2 n$. This property is needed since any constraint $x_{i}-x_{j} \leq \alpha$, $1 \leq i, j \leq n$ can be represented as both $y_{2 i-1}-y_{2 j-1} \leq \alpha$ and $y_{2 j}-y_{2 i} \leq \alpha$. If $m$ is coherent, we denote by

$$
\gamma^{O c t}(m)=\left\{\left(v_{1}, v_{2}, \ldots, v_{n}\right) \in \mathbb{Z}^{n} \mid\left(v_{1},-v_{1}, v_{2},-v_{2}, \ldots, v_{n},-v_{n}\right) \in \gamma(m)\right\}
$$

the set of concretizations, i.e. the set of models of the octagonal constraint represented by $m$. Also, $m$ is said to be consistent if $\gamma^{\text {Oct }}(m) \neq \emptyset$, and inconsistent otherwise. The octagonal graph of an octagonal constraint $\phi(\mathbf{x})$, represented as a difference bound constraint $\bar{\phi}(\mathbf{y})$ with DBM $m$, has vertices $\mathbf{y}$, and an arc labeled by $m_{i, j}$ between $y_{i}$ and $y_{j}$ if and only if $m_{i, j}<\infty, 1 \leq i, j \leq 2 n$. 
Definition 9. A consistent coherent $2 n \times 2 n D B M m$ in $\mathbb{Z}$ is said to be tightly closed if and only if the following hold:

1. $m_{i, i}=0 \forall 1 \leq i \leq 2 n$

2. $m_{i, \bar{i}}$ is even $\forall 1 \leq i \leq 2 n$

3. $m_{i, j} \leq m_{i, k}+m_{k, j} \forall 1 \leq i, j, k \leq 2 n$

4. $m_{i, j} \leq\left(m_{i, \bar{i}}+m_{\bar{j}, j}\right) / 2 \forall 1 \leq i, j \leq 2 n$

The intuition behind the last point is the following: since $y_{i}-y_{\bar{i}} \leq m_{i, \bar{i}}$ and $y_{\bar{j}}-y_{j} \leq m_{\bar{j}, j}$ and $y_{i}=-y_{\bar{i}}, y_{j}=-y_{\bar{j}}$ if we interpret the DBM $m$ as an octagonal constraint, we have that $y_{i} \leq\left\lfloor\frac{m_{i, \bar{i}}}{2}\right\rfloor$ and $-y_{j} \leq\left\lfloor\frac{m_{\bar{j}, j}}{2}\right\rfloor$, hence $y_{i}-y_{j} \leq$ $\left\lfloor\frac{m_{i, \bar{i}}}{2}\right\rfloor+\left\lfloor\frac{m_{\bar{j}, j}}{2}\right\rfloor$. Therefore, the tightening of an octagonal DBM has to come up with values for $m_{i, j}$ that are smaller than $\left\lfloor\frac{m_{i, \bar{i}}}{2}\right\rfloor+\left\lfloor\frac{m_{\bar{j}, j}}{2}\right\rfloor$, for all $1 \leq i, j \leq 2 n$.

The tight closure of a $2 n \times 2 n$ octagonal DBM $m$ is an octagonal DBM $m_{t}^{*}$ such that $\gamma^{O c t}(m)=\gamma^{O c t}\left(m_{t}^{*}\right)$, and $m_{t}^{*}$ is tightly closed. By Theorem 7 in [15], we know that the tight closure of a consistent octagonal DBM exists and is unique.

We now characterize the consistency of octagonal constraints using their DBM representations. The following result, proved in [2], is crucial for the developments of the next section, therefore we cite (a slightly modified version of) it here:

Theorem 1 ([2]). Let $m$ be a $2 n \times 2 n$ octagonal DBM consistent and coherent, and let $m^{*}$ be its closure. Suppose that $\left\lfloor\frac{m_{i, \bar{i}}^{*}}{2}\right\rfloor+\left\lfloor\frac{m_{i, i}^{*}}{2}\right\rfloor \geq 0$, for all $1 \leq i \leq 2 n$. Then $\gamma^{\text {Oct }}(m) \neq \emptyset$ and the DBM $m^{T}$ defined as:

$$
m_{i, j}^{T}=\min \left\{m_{i, j}^{*},\left\lfloor\frac{m_{i, \bar{i}}^{*}}{2}\right\rfloor+\left\lfloor\frac{m_{\bar{j}, j}^{*}}{2}\right\rfloor\right\}
$$

for all $1 \leq i, j \leq 2 n$, is the tight closure of $m$.

It follows immediately that an octagonal constraint is consistent if and only if (1) its constraint graph representation does not contain negative weight cycles and moreover, (2) if the sum between the halved weights of the minimal paths from any node $x_{i}$ to $x_{\bar{i}}$, and from $x_{\bar{i}}$ and $x_{i}$ is positive. Notice that the lack of negative weight cycles alone is not enough, as shown by the following example.

Example 2. Let $\phi\left(x, y, z, x^{\prime}, y^{\prime}, z^{\prime}\right)=\left(x-y^{\prime} \leq 1\right) \wedge\left(y^{\prime}+x \leq-2\right) \wedge\left(-x+z^{\prime} \leq\right.$ 1) $\wedge\left(-z^{\prime}-x \leq 0\right)$ be an octagonal constraint on integer variables $x, y, z$ and $x^{\prime}, y^{\prime}, z^{\prime}$. The constraint is inconsistent since it implies $(-1 \leq 2 x \leq-1)$ and thus has no integer solution. However, its corresponding octagonal DBM does not contain negative weight cycles. The tightening step, i.e. replacing each element $m_{i \bar{i}}$ with $2\left\lfloor\frac{m_{i \bar{i}}}{2}\right\rfloor$, exhibits two negative weight cycles, between $+x$ and $-x$, and between $+z^{\prime}$ and $-z^{\prime}$.

We are now ready to give the result of existential quantification over variables occurring within octagons. Namely, we prove that, if $\phi\left(x_{1}, \ldots, x_{n}\right)$ is an octagonal constraint, the formula $\exists x_{k}$. $\phi$, for some $1 \leq k \leq n$ is again an octagonal 
constraint, and its representation is effectively computable from the constraint graph of $\phi$.

In the following, we assume w.l.o.g. that $k=n$ (if this is not the case, we proceed to reindexing variables). From now on assume that $\phi\left(x_{1}, \ldots, x_{n}\right)$ is consistent, and let $m$ be its $2 n \times 2 n$ tightly closed coherent octagonal DBM. We denote by $\beta(m)$ the $2 n-2 \times 2 n-2$ matrix from which the $2 n-1$ and $2 n$ lines and columns have been eliminated. Notice that these correspond to the $x_{n}$ and $-x_{n}$ terms, respectively.

Lemma 1. Let $m \in \mathbb{Z}_{2 n \times 2 n}$ be the coherent and tightly closed difference bound matrix for $\phi\left(x_{1}, \ldots, x_{n}\right)$. Then $\beta(m)$ is also coherent and tightly closed.

The following theorem proves that octagons are closed under existential quantification.

Theorem 2. Let $\mathbf{x}=\left(x_{1}, x_{2}, \ldots, x_{n}\right)$ be a set of variables ranging over $\mathbb{Z}, \phi(\mathbf{x})$ a consistent octagonal constraint and $m$ the corresponding $2 n \times 2 n$ octagonal $D B M$. Then formula $\exists x_{k} . \phi(\mathbf{x})$ is equivalent to erasing vertices $y_{2 k}$ and $y_{2 k-1}$ together with the incident arcs from the graph $\mathcal{G}\left(m_{t}^{*}\right)$. Moreover, the resulting graph is also coherent and tightly closed.

\section{Octagonal Relations}

This section is dedicated to our main result, the definition of the transitive closure of an octagonal relation in Presburger arithmetic. An octagonal relation is defined in a similar way to a difference bound relation.

Definition 10. Let $\mathbf{x}=\left(x_{1}, x_{2}, \ldots, x_{n}\right), \mathbf{x}^{\prime}=\left(x_{1}^{\prime}, x_{2}^{\prime}, \ldots, x_{n}^{\prime}\right)$ be sets of variables ranging over $\mathbb{Z}$. Then an octagonal relation $R\left(\mathbf{x}, \mathbf{x}^{\prime}\right)$ is a relation that can be written as a finite conjunction of terms of the form $\pm x_{i} \pm x_{j} \leq a_{i, j}, \pm x_{i}^{\prime} \pm x_{j} \leq$ $b_{i, j}, \pm x_{i} \pm x_{j}^{\prime} \leq c_{i, j}, \pm x_{i}^{\prime} \pm x_{j}^{\prime} \leq d_{i, j}, \pm 2 x_{i} \leq e_{i, j}$ or $\pm 2 x_{i}^{\prime} \leq f_{i, j}$, where $a_{i, j}, b_{i, j}, c_{i, j}, d_{i, j}, e_{i, j}, f_{i, j} \in \mathbb{Z}$ and $1 \leq i, j \leq n, i \neq j$.

The DBM and graph representation of an octagonal relation are defined as in the previous case of difference bound relations. For the rest of this section, let $R\left(\mathbf{x}, \mathbf{x}^{\prime}\right)$ be an octagonal relation and $\bar{R}\left(\mathbf{y}, \mathbf{y}^{\prime}\right)$ be its difference bound representation. Let $\mathcal{G}$ be the graph of $\bar{R}, \bar{R}^{k}$ be the $k$-th iteration of $\bar{R}, m_{k}$ be its DBM, and $\mathcal{G}^{k}$ be the graph corresponding to $\bar{R}^{k}$, obtained by connecting $k$ copies of $\mathcal{G}$. The formal definition of $\mathcal{G}^{k}$ is similar to Definition 6 .

In order to compute $R^{k}$, we need two ingredients. First, we need to check for consistency of the unfolded relation, that is we need to check that $\gamma^{O c t}\left(m_{k}\right) \neq \emptyset$. Second, we need to obtain the strongest octagonal constraints between $\mathbf{y}^{(\mathbf{0})}$ and $\mathbf{y}^{(\mathbf{k})}$ in $\mathcal{G}^{k}$. By Theorem 2 all intermediate vertices $\mathbf{y}^{(\mathbf{l})}, 0<l<k$ can be eliminated from $\mathcal{G}^{k}$, and the result is a tightly closed octagonal graph, whose interpretation is equivalent to $R^{k}$.

By Theorem 1, both points require the computation of the values $\left(m_{k}{ }^{*}\right)_{i^{(l)}, \overline{i^{(l)}}}$ and $\left(m_{k}{ }^{*}\right)_{i^{(l)}, i^{(l)}}$, for all $1 \leq i \leq 2 n$ and $0 \leq l \leq k$, where the index $i^{(l)}$ refers to 


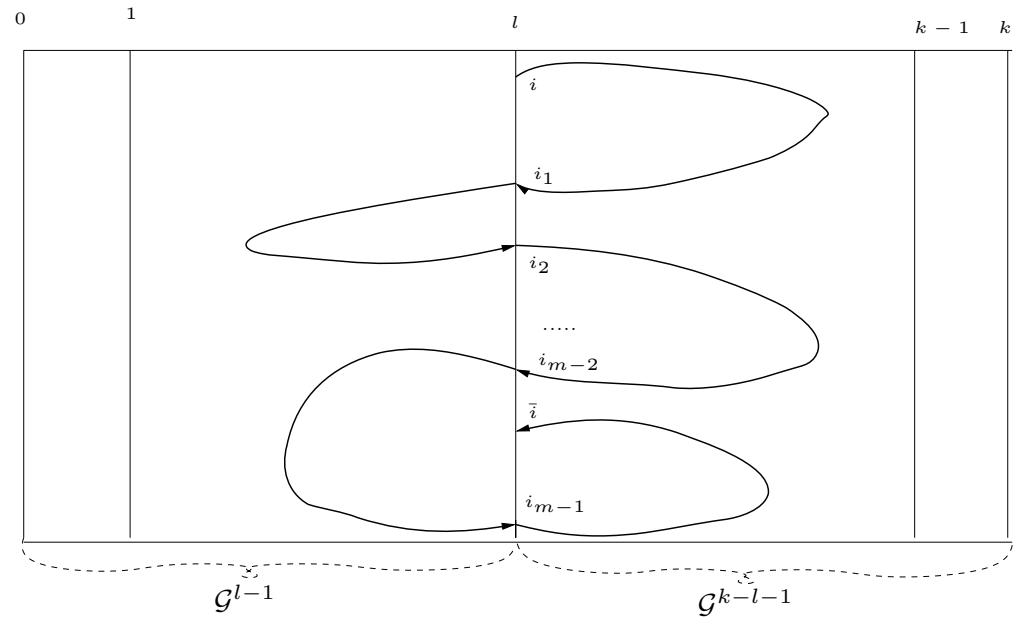

Fig. 2. Computing paths of weight $\left(m_{k}^{*}\right)_{i(l)}, \overline{i(l)}$

the variable $y_{i}^{(l)}$ in the DBM representation of $\bar{R}\left(\mathbf{y}, \mathbf{y}^{\prime}\right)$. But these values can now be defined by Presburger formulae, using the results from [76]. To understand this point, consider the situation depicted in Figure 2 .

Assume in the following that $\mathcal{G}^{k}$ has no cycles of negative weight. The absence of negative cycles can be checked a-priori using e.g. the method in [6]. Since we are aiming at computing minimal weight paths, it is sufficient to consider acyclic paths only 4 . For a fixed $0<l<k$, an acyclic path between the nodes $y_{i}{ }^{(l)}$ and $y_{\bar{i}}{ }^{(l)}$, for some $1 \leq i \leq 2 n$, can be decomposed in at most $2 n-1$ segments starting and ending in $\mathbf{y}^{(l)}$, but not intersecting with $\mathbf{y}^{(l)}$, other than in the beginning and in the end. Moreover, if the path considered is of minimal weight, these segments are of minimal weight as well.

Let $1 \leq i=i_{0}, i_{1}, \ldots, i_{m}=\bar{i} \leq 2 n$, be a set of pairwise distinct indices, such that

$$
\left(m_{k}^{*}\right)_{i^{(l)}, \overline{i^{(l)}}}=\sum_{j=0}^{m-1}\left(m_{k}^{*}\right)_{i_{j}^{(l)}, i_{j+1}^{(l)}}
$$

As in Figure 2] several of the paths $y_{i}^{(l)} \leadsto y_{i_{1}}^{(l)}, y_{i_{1}}^{(l)} \leadsto y_{i_{2}}^{(l)}, \ldots, y_{i_{m-1}}^{(l)} \leadsto y_{\bar{i}}^{(l)}$ will contain only nodes from the set $\mathbf{y}^{(\leq l)}$, whereas the rest will contain only nodes from the set $\mathbf{y}^{(\geq l)}$. Notice that, the paths from $\mathbf{y}^{(\leq l)}$ connect the terminal nodes of $\mathcal{G}^{l-1}$, whereas the paths from $\mathbf{y}^{(\geq l)}$ connect the initial nodes of $\mathcal{G}^{k-l-1}$. Therefore, we have:

$$
\begin{aligned}
& -\left(m_{k}^{*}\right)_{i_{j}^{(l)}, i_{j+1}^{(l)}}=\min _{l-1}\left\{y_{i_{j}}^{(l)} \leadsto y_{i_{j+1}}^{(l)}\right\} \text {, if } y_{i_{j}}^{(l)} \leadsto y_{i_{j+1}}^{(l)} \text { belongs to } \mathbf{y}^{(\leq l)}, \text { and } \\
& -\left(m_{k}^{*}\right)_{i_{j}^{(l)}, i_{j+1}^{(l)}}=\min _{k-l-1}\left\{y_{i_{j}}^{(0)} \leadsto y_{i_{j+1}}^{(0)}\right\}, \text { if } y_{i_{j}}^{(l)} \leadsto y_{i_{j+1}}^{(l)} \text { belongs to } \mathbf{y}^{(\geq l)} .
\end{aligned}
$$

\footnotetext{
${ }^{4}$ If a path has a cycle of a positive weight, it cannot be minimal.
} 
But since the $\min _{j}\{x \leadsto y\}$ functions are definable in Presburger arithmetic [76], we obtain that $\left(m_{k}^{*}\right)_{i, \bar{i}}$ are Presburger definable as well. Since moreover, integer division can be defined in Presburger as

$$
\left\lfloor\frac{u}{2}\right\rfloor=v \Longleftrightarrow 2 v \leq u \leq 2 v+1
$$

it is possible to encode the consistency check of Theorem 1 by a Presburger formula, and effectively perform the check required by Theorem 1:

$$
\bigwedge_{\substack{1 \leq i \leq 2 n \\ 0 \leq l \leq k}}\left\lfloor\frac{m_{i^{(l)}, \overline{i^{(l)}}}^{*}}{2}\right\rfloor+\left\lfloor\frac{m_{i^{(l)}}^{*}, i^{(l)}}{2}\right\rfloor \geq 0
$$

The formula used to check consistency of $R\left(\mathbf{x}, \mathbf{x}^{\prime}\right)^{k}, k \geq 0$ is of size $\mathcal{O}\left(2^{2 n \log 2 n}\right)$, where $n$ is the number of variables in $\mathbf{x}$. For more details, the interested reader is pointed to 4 .

After quantifier elimination, the strongest octagonal relations are obtained by taking the restriction of the tight closure of $m_{k}$ to $\mathbf{y}^{(0)}$ and $\mathbf{y}^{(k)}$, according to Theorem 1 and Theorem 2 .

$$
\begin{aligned}
& y_{i}{ }^{(0)}-y_{j}{ }^{(k)} \leq\left(m_{k t}^{*}\right)_{i(0), j^{(k)}}=\min \left\{\left(m_{k t}^{*}\right)_{i^{(0)}, j^{(k)}},\left\lfloor\frac{\left(m_{k t}^{*}\right)_{i(0)} \overline{i^{(0)}}}{2}\right\rfloor+\left\lfloor\frac{\left(m_{k t}^{*}\right) \bar{j}^{(k)}, j^{(k)}}{2}\right\rfloor\right\} \\
& y_{i}{ }^{(k)}-y_{j}{ }^{(0)} \leq\left(m_{k t}^{*}\right)_{i(k), j^{(0)}}=\min \left\{\left(m_{k t}^{*}\right)_{i(k), j^{(0)}},\left\lfloor\frac{\left(m_{k t}^{*}\right)_{i^{(k)}, \overline{i^{(k)}}}}{2}\right\rfloor+\left\lfloor\frac{\left(m_{k t}^{*}\right)_{\overline{j^{(0)}}, j^{(0)}}}{2}\right\rfloor\right\}
\end{aligned}
$$

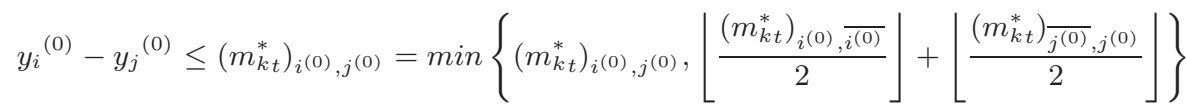

$$
\begin{aligned}
& y_{i}{ }^{(k)}-y_{j}{ }^{(k)} \leq\left(m_{k t}^{*}\right)_{i(k), j(k)}=\min \left\{\left(m_{k t}^{*}\right)_{i(k), j^{(k)}},\left\lfloor\frac{\left(m_{k t}^{*}\right)_{i^{(k)}, \overline{i^{(k)}}}}{2}\right\rfloor+\left\lfloor\frac{\left(m_{k t}^{*}\right)_{j^{(k)}, j^{(k)}}}{2}\right\rfloor\right\}
\end{aligned}
$$

Since we showed that all weights of the form $m_{i^{(l)}, \overline{i(l)}}^{*}$ are definable in Presburger arithmetic, it follows that $R^{k}$ is also Presburger definable. Consequently, the transitive closure of $R$ is the Presburger formula $\exists k \cdot R^{k}$, leading to the following theorem:

Theorem 3. The transitive closure of an integer octagonal relation is Presburger definable.

Example 3. Let $R\left(\mathbf{x}, \mathbf{x}^{\prime}\right)$ be the following octagonal relation, where $\mathbf{x}=\left\{x_{1}, x_{2}\right\}$

$$
R\left(\mathbf{x}, \mathbf{x}^{\prime}\right)=x_{1}+x_{2} \leq 5 \wedge x_{1}^{\prime}-x_{1} \leq-2 \wedge x_{2}^{\prime}-x_{2} \leq-3
$$

Figure 3 shows the graph representation of $R^{k}$ for $k=1,2,3, \ldots$. The $n$-step closure is:

$$
\begin{aligned}
R^{n}\left(\mathbf{x}, \mathbf{x}^{\prime}\right)= & x_{1}+x_{2} \leq 5 \wedge x_{1}^{\prime}-x_{1} \leq-2 n \wedge x_{2}^{\prime}-x_{2} \leq-3 n \wedge \\
& x_{1}+x_{2}^{\prime} \leq 5-3 n \wedge x_{2}+x_{1}^{\prime} \leq 5-2 n \wedge x_{1}^{\prime}+x_{2}^{\prime} \leq 5-5 n
\end{aligned}
$$



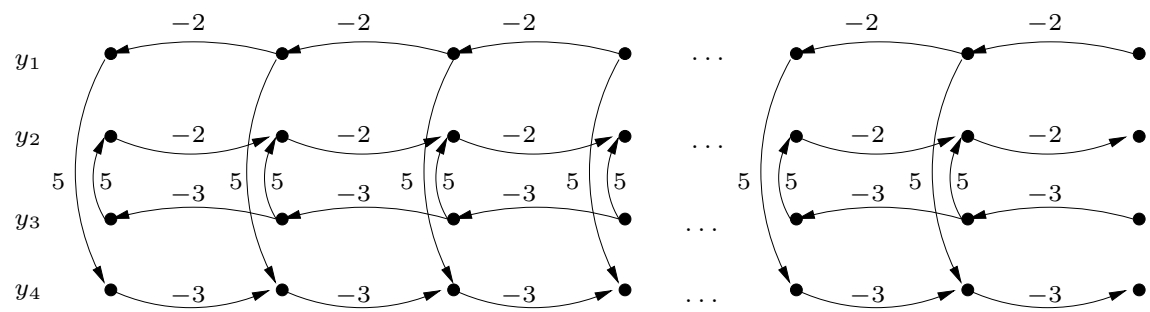

Fig. 3. Graph representation of $\bar{R}^{k}, k=1,2,3, \ldots$

\section{Implementation and Experience}

We have implemented the method for computing transitive closures of octagonal relations in a tool for the analysis of counter automata, that we are currently

Table 1. Experimental results

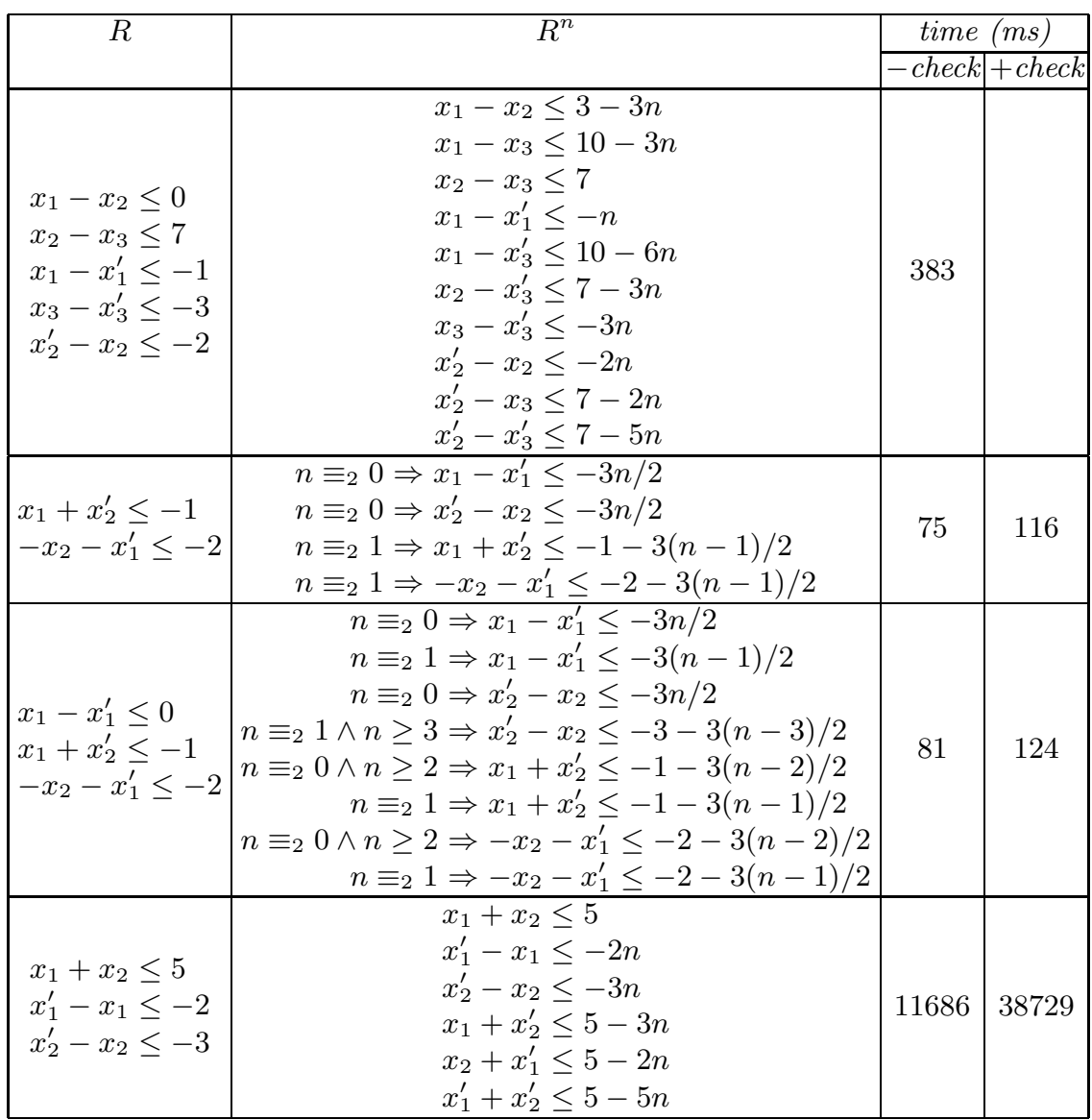


developing. Table 1 shows several octagonal relations (first column), the closed form of their iteration (second column), the execution times (in milliseconds) needed to compute the closed forms without consistency checks (third column), and with consistency checks (fourth column). Notice that the first relation is the difference bound relation example from Section 2, for which no consistency check is needed.

The tool, called FLATA, is implemented in Java $(\mathrm{TM})$, and it is currently available at 10. The execution times from Table 1 are relative to a Intel $^{(\mathrm{R})}$ Xeon ${ }^{(\mathrm{TM})}$ CPU 3.00GHz equipped with $1 \mathrm{~Gb}$ of RAM. As an approximate upper bound, we can in principle handle difference bound relations on ten counters, and/or octagons on five counters, within reasonable execution times ( $\approx 10 \mathrm{~min})$.

The fourth example from Table 1 takes significantly more time than the previous ones. This happens because in the pre-processing step we need to replace $x_{1}+x_{2} \leq 5$ with $x_{1}-x_{3}^{\prime} \leq 5 \wedge x_{3}^{\prime}+x_{2} \leq 0$, where $x_{3}$ is a fresh variable, thus having to deal with a DBM relation with 6 counters.

\section{Conclusions}

We have considered the problem of computing transitive closures of octagonal relations, which are finite conjunctions of atomic formulae of the form $\pm x \pm y \leq c$, where $x$ and $y$ are possibly primed integer variables. We show that the $k$-th iteration of such a relation has a Presburger definable closed form. As a consequence, the transitive closure is also Presburger definable. This result enlarges the class of non-deterministic counter automata for which the reachability problem is decidable, to counter automata with octagonal transition relations. This result is expected to have an impact in the fields of software verification, as abstract models of software systems are described using octagons. We have implemented our method in a tool for the analysis of counter automata, and report on a number of experiments.

\section{References}

1. Bagnara, R.: Data-Flow Analysis for Constraint Logic-Based Languages. Ph. D. Thesis, Dipartimento di Informatica, Università di Pisa (1997)

2. Bagnara, R., Hill, P.M., Zaffanella, E.: An improved tight closure algorithm for integer octagonal constraints. In: Logozzo, F., Peled, D.A., Zuck, L.D. (eds.) VMCAI 2008. LNCS, vol. 4905, pp. 8-21. Springer, Heidelberg (2008)

3. Boigelot, B.: On iterating linear transformations over recognizable sets of integers. TCS 309(2), 413-468 (2003)

4. Bozga, M., Girlea, C., Iosif, R.: Iterating octagons. TR VERIMAG (2008)

5. Bozga, M., Habermehl, P., Iosif, R., Vojnar, T.: A logic of singly indexed arrays. In: LPAR 2008. LNCS(LNAI), vol. 5330, pp. 558-573. Springer, Heidelberg (2008)

6. Bozga, M., Iosif, R., Lakhnech, Y.: Flat parametric counter automata. In: Bugliesi, M., Preneel, B., Sassone, V., Wegener, I. (eds.) ICALP 2006. LNCS, vol. 4052, pp. 577-588. Springer, Heidelberg (2006) 
7. Comon, H., Jurski, Y.: Multiple counters automata, safety analysis and presburger arithmetic. In: Vardi, M. (ed.) CAV 1998. LNCS, vol. 1427, pp. 268-279. Springer, Heidelberg (1998)

8. Cousot, P., Halbwachs, N.: Automatic discovery of linear restraints among variables of a program. In: POPL, pp. 84-97. ACM Press, New York (1978)

9. Finkel, A., Leroux, J.: How to compose presburger-accelerations: Applications to broadcast protocols. In: Agrawal, M., Seth, A.K. (eds.) FSTTCS 2002. LNCS, vol. 2556, pp. 145-156. Springer, Heidelberg (2002)

10. http://www-verimag.imag.fr/ async/flata/flata.html

11. Habermehl, P., Iosif, R., Vojnar, T.: What else is decidable about integer arrays? In: Amadio, R. (ed.) FOSSACS 2008. LNCS, vol. 4962, pp. 474-489. Springer, Heidelberg (2008)

12. Harvey, W., Stuckey, P.: A unit two variable per inequality integer constraint solver for constraint logic programming. In: Australian Computer Science Conference, pp. 102-111 (1997)

13. Ibarra, O.H.: Reversal-bounded multicounter machines and their decision problems. Journal of the ACM 25(1), 116-133 (1978)

14. Leroux, J., Sutre, G.: Flat counter automata almost everywhere? In: Peled, D.A., Tsay, Y.-K. (eds.) ATVA 2005. LNCS, vol. 3707, pp. 489-503. Springer, Heidelberg (2005)

15. Miné, A.: The octagon abstract domain. Higher-Order and Symbolic Computation 19(1), 31-100 (2006)

16. Minsky, M.: Computation: Finite and Infinite Machines. Prentice-Hall, Englewood Cliffs (1967)

17. Presburger, M.: Über die Vollstandigkeit eines gewissen Systems der Arithmetik. In: Comptes rendus du I Congrés des Pays Slaves, Warsaw (1929)

18. Reutenauer, C.: Aspects Mathématiques des Réseaux de Petri. Collection Études et Recherches en Informatique. Masson (1989) 